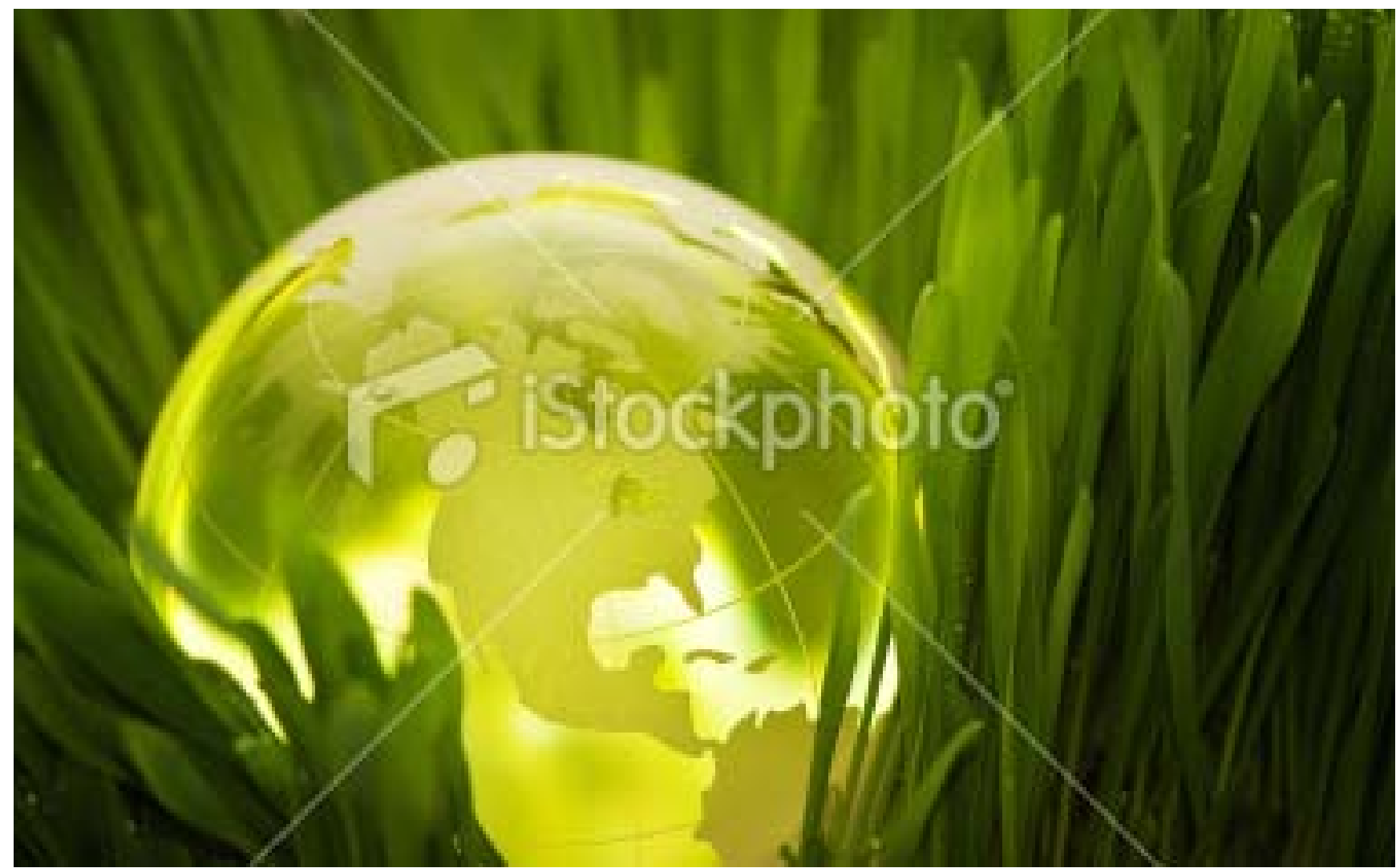

\title{
Customer Value und Public Value Der Kundennutzen im Bauch der Gesellschaft
}

Die exponentiell zunehmende Anzahl von Kundensegmenten, Kundenbedürfnissen und Kundeninteraktionspunkten zählt heute zu den größten Herausforderungen des Marketing. Spiegelbild dieser steigenden Komplexität ist die grundsätzliche Frage der Beziehung zur Gesellschaft, d. h. die Frage nach der „Public Value Proposition“ im Gegensatz zu einer immer weiter differenzierten „Customer Value Proposition“. Die Autoren stellen in ihrem Beitrag inhaltliche Querbezüge zwischen Customer Value und Public Value her und reflektieren über Marketingaktivitäten als Medium eines gesellschaftlichen Dialogs über Sinn- und Wertvorstellungen.

$\mathrm{D}$ ie konsequente Ausrichtung auf den subjektiv erlebten Kundennutzen ist ein zentrales Thema eines erfolgreichen Marketing: Nutzen und damit Wert für den Kunden entsteht schließlich nur in der Erfah-rungswelt des Kunden (Belz/Bieger 2004). Dabei ist auch der soziale Aspekt dieser Erfahrungswelt bedeutsam, wenn etwa im Rahmen einer Premium-Strategie bestimmte Produkte oder Marken mit „Prestige“ aufgeladen werden (Kotler 2000). Nur:
Mit dem Kunden sitzt immer auch die Gesellschaft am Tisch. Denn ebenso wie jedes Angebot auf ein aus einem sozialen Zusammenhang erwachsendes Bedürfnis reagiert, wirkt es über den einzelnen Kunden hinaus darauf zurück.

Modernes Marketing aktiviert, nutzt und moderiert also gerade das Spannungsfeld zwischen Einzelnem und sozialem Umfeld, zwischen Individualität und Gruppenzugehörigkeit. Umgekehrt sind Marketingaktivi- täten damit aber auch sozialen Maßstäben unterworfen: Hat individueller Kundennutzen negative Externalitäten zur Folge, führt also das Kundenverhalten zu einer Minderung des Gemeinwohls, kann dies eine gesellschaftliche Debatte auslösen - wie etwa zur Frage, ob der individuelle Nutzen des Autofahrens dem gesamtgesellschaftlichen Interesse an Ressourcenschonung vorgeht. Dadurch bilden sich gesellschaftliche Normen, Werte und Regeln heraus, die der „un- 
sichtbaren Hand" einen Rahmen stecken. Abbildung 1 illustriert schematisch den Kreislauf.

Nutzen für den Kunden stiften heißt heute also, mehr denn je in eine gesellschaftliche „Werte“-Dynamik eingreifen. Marketing tritt damit in die gesellschaftliche Sphäre ein. Neu ist heute das Ausmaß an zu bewältigender Komplexität. Während in den letzten Jahren die Thematik unter Stichworten, wie z. B. „nachhaltiges Marketing“, „politischer Konsum“, oder „Issue Management“ behandelt wurden, sind wir der Auffassung, dass ein Produkt- und Dienstleistungsangebot in hochdynamischen Prozessen mehr denn je auf gesellschaftliche Wirkungen zu prüfen und als Teil eines Gemeinwesens zu verstehen ist. Das Neue oder besser, die wieder stärker in den Vordergrund rückende Herausforderung' besteht darin, den Customer Value auch als mehrdimensionale gesellschaftliche Wertschöpfung (Public Value) zu begreifen.

\section{Moralisierte Märkte und beschleunigte Gesellschaft als Treiber}

Marketing ist „die bewusst marktorientierte Führung des gesamten Unternehmens, die sich in Planung, Koordination und Kontrolle aller auf die aktuellen und potenziellen Märkte ausgerichteten Unternehmensaktivitäten niederschlägt" (Meffert 2007). Bei marktorientierter Unternehmensführung stehen die Wünsche und Bedürfnisse aktueller und potenzieller Kunden am Anfang aller Überlegungen (Meffert 2007). Jedoch sind „die“

\section{„Mit den Kunden sitzt immer auch die Gesellschaft am Tisch.“}

dem engeren Kreis von Vertriebspartnern, Konkurrenten etc. gewinnt dabei eine kritische Öffentlichkeit an Bedeutung. Nach Stehr (2007) bewirken die Zunahme des Wohlstands und des verfügbaren Wissens eine „Moralisierung der Märkte“, indem etwa die Art und Weise der Herstellungsbedingungen, der Fertigungsprozesse oder des Marktauftritts für die Kunden transparent und zu einem wesentlichen Bewertungskriterium werden. Zugleich erlauben es die heutigen Kommunikationsmöglichkeiten den Kunden- oder Interessensgruppen, mit geringem Aufwand Informationen zu sammeln und zu verbreiten oder ihre Interessen zu bündeln - zum Beispiel gelang es einer Gruppe kanadischer Kleinanleger, sich über ein Weblog auf „Facebook“ zu organisieren, um gegen einen für sie unvorteilhaften Ausgang eines Insolvenzverfahrens $\mathrm{zu}$ streiten (Economist, 2008). Diese zunehmende Transparenz

Kunden immer schwerer zu greifen: Insbesondere im Konsumgüterbereich steht der „verschwindenden Mitte“ eine explosionsartige Zunahme von Kundensegmenten, Vertriebskanälen und Kundeninteraktionspunkten gegenüber (Court 2006).

Hinzu kommt, dass Unternehmen neben dem Kunden als Käufer auch andere externe Gruppen betrachten müssen - neben bei gleichzeitig höheren moralischen Ansprüchen des „politischen“ oder „ethischen“ Kunden (z. B. Harrison/Newholm/Shaw 2005) erfordert auf Unternehmensseite offenkundig eine höhere Sensibilität und mehr denn je vernetztes, ganzheitliches Denken (Gomez/Probst 1999).

In der bisher geltenden Sicht wurde empfohlen, die einzelnen Stakeholder differen- ziert zu beachten (Freeman 1984). Das wird allerdings immer schwieriger, wenn die Gruppe der Stakeholder in einer globalisierten (Medien-)Welt ständig anwächst, immer heterogener wird, sich dynamisch verändert, während möglicherweise auch inkompatible Ansprüche und schwer analysierbare Wechselwirkungen vorhanden sind (Walsh 2005). Einer weiteren Verfeinerung der Analysetechniken sind prinzipielle Erkenntnisgrenzen gesetzt, so dass Als-ob-Annahmen, fiktionale Bezugnahmen, Werte-Wetten unumgänglich werden (Meynhardt 2004).

Hinzu kommt eine Tendenz der sozialen Beschleunigung (Rosa 2005) als zweiter Grund, warum Marketingaktivitäten stärker in die gesellschaftliche Wertekommunikation „hineingezogen“ werden. Bedürfnissen und Wünschen der Kunden gerecht zu werden, bedeutet in der Erfahrungswelt des Kunden sinnstiftend zu wirken. In einem durch hohe Instabilitäts- und Wandelraten beschreibbaren gesellschaftlichen Umfeld besteht ein hohes Maß an Orientierungs-, Anpassungs- und Lernbedarf. Ein durch Erfahrungen der Beschleunigung wirtschaftlicher Prozesse, des Lebenstempos und des sozialen Wandels ausgelöster permanenter Abstimmungsbedarf zwischen unterschiedlichen Teilsystemen der Gesellschaft und Lebenswelten eröffnet neue Möglichkeiten der Um- und Neubewertung. In einer als zukunftsoffen empfunde- 
Tab. 1 Zusammenhang Grundbedürfnisse und Wertdimension

\begin{tabular}{|l|l|l|}
\hline Grundbedürfnis & Relevante Bewertungsprozesse & Wertdimension \\
\hline $\begin{array}{l}\text { Orientierung und Kontrolle } \\
\text { (Streben nach Regelhaftigkeiten und } \\
\text { eigenem Handlungsspielraum) }\end{array}$ & $\begin{array}{l}\text { - Verhältnis zwischen Aufwand und } \\
\text { Nutzen } \\
\text { "Hilfestellung" bzw. Beitrag zur } \\
\text { Problemlösung } \\
\text { - Kontroll- und Einflussmöglichkeiten }\end{array}$ & Instrumentell-utilitaristisch \\
\hline $\begin{array}{l}\text { Selbstwerterhalt und -erhöhung } \\
\text { (Streben nach positivem Selbstbild und } \\
\text { stimmigem Person-Umweltbezug) }\end{array}$ & $\begin{array}{l}\text { - Selbstwertgefühl } \\
\text { - Individualität/Autonomie } \\
\text { - Gerechtigkeit und Gleichbehandlung }\end{array}$ & Moralisch-ethisch \\
\hline $\begin{array}{l}\text { Bindung } \\
\text { (Streben nach positiven Beziehungs- } \\
\text { erfahrungen) }\end{array}$ & $\begin{array}{l}\text { - Gruppenidentifikation } \\
\text { - Statusempfinden } \\
\text { - Soziale Differenzierung }\end{array}$ & Politisch-sozial \\
\hline $\begin{array}{l}\text { Positive Erfahrungen } \\
\text { (Streben nach positiven Emotionen) }\end{array}$ & $\begin{array}{l}\text { - Lust-/Unlusterfahrung } \\
\text { - Genusserleben } \\
\text { - Schönheit }\end{array}$ & Hedonistisch-ästhetisch \\
\hline
\end{tabular}

nen Gesellschaft mit einem „progressiven Verlust der politischen Selbsteinwirkungsmöglichkeiten der Gesellschaft" (Rosa 2005, S. 333) kommt dabei dem dezentralen Aushandeln von Werten und Normen eine größere Bedeutung zu. Auch Marketingaktivitäten werden ,anfälliger“ für unvorhersehbare Bewertungsgesichtspunkte im gesellschaftlichen Umfeld.

Öffentlich sensible Bereiche wie Umweltschutz, Qualität von Lebensmitteln oder medizinischer Fortschritt betreffen neben dem Einzelnen die Gesellschaft als Ganzes so grundlegend, dass hierbei Ängste, Hoffnungen, Meinungen, wahrgenommene Fairness und Nachhaltigkeit, Vermutungen usw. in hohem Maße etwa die Reputation eines Produktes oder eines Unternehmens und damit auch Kaufentscheidungen prägen. Folglich wird die Frage nach übergeordneten menschlichen Grundbedürfnissen wichtiger als eine noch weitere Ausdifferenzierung von Zusatzund Nebennutzen. Grundlegende Fragen wie „Was ist unser Auftrag? Wie erreichen wir gesellschaftliche Zustimmung zu unseren Aktivitäten? Wie begründen wir unsere License to Operate?" müssen aus Unternehmenssicht viel stärker von innen heraus beantwortet werden. Je unübersichtlicher die Lage, desto relevanter wird die nicht in die Marktlogik von Angebot und Nachfrage delegierbare Frage nach einer grundlegenden „Value Proposition“, die sich konsequent an gesellschaftlichen Erwartungen messen lässt.

\section{Von der Gesellschaft her denken}

Für Unternehmen bietet dies erhebliche Chancen, durch die Interpretation sich ändernder Werte und Normen neue Bedürfnisse zu identifizieren und zu befriedigen. Andererseits bedeutet dies auch, dass eigenes Handeln vor dem Hintergrund vielfältiger und sich ändernder Kriterien bewertet wird. Wer im gesellschaftlichen Diskurs verhandelte „Werte“, wie z.B. Umgang mit dem Alter, Geschlechterrollen, Sicherheitsempfinden, Ernährungsverhalten zum Thema seines Leistungssystems macht, begibt sich direkt in den gesellschaftlichen Dialog hinein. Und je weiter die „Moralisierung " der Märkte geht, desto schneller können Unternehmen auch ungewollt in den gesellschaftlichen Dialog eingebunden werden.

Es ist deshalb im eigenen Interesse des Unternehmens, neben eine Analyse des Kundennutzens auch eine des gesellschaftlichen Umfeldes zu stellen. In dieser Sicht kommt der eigenen Value Proposition als selbstdefiniertem Auftrag eine bedeutsame Rolle zu, denn die Wertschöpfung ist immer mit dem gesellschaftlichen Umfeld verknüpft: Zum Beispiel sind unternehmerische Aktivitäten nur dort möglich, wo ein Mindestmaß an Eigentumsrechten existiert und auch durchsetzbar ist. Diese „selbst- verständlichen“ Eigentumsrechte sind jedoch ihrerseits das Ergebnis eines gesellschaftlichen Konsenses. Die Grundlagen der sozialen Marktwirtschaft sind keineswegs axiomatisch und daher auch keineswegs selbstverständlich akzeptiert (Köcher 2006). Vielmehr bedürfen sie ihrerseits der moralischen Rechtfertigung und öffentlicher Akzeptanz: Sicherlich dürfen forschende Arzneimittelunternehmen die Früchte ihrer Arbeit durch Patente schützen. Aber sind nicht auch Entwicklungsländer „im Recht“, wenn sie genau diese $\mathrm{Pa}$ tente verletzen, um ihre Bevölkerung mit Medikamenten zu versorgen?

Gleiches gilt für die mit vielen unternehmerischen Aktivitäten verbundenen externen Effekte, deren Legitimierung (oder Beseitigung) eine kritische Öffentlichkeit einfordert.

Der zu erschaffende Customer Value wird also vor dem Hintergrund eines $\mathrm{Pu}$ blic Value als eine gesellschaftliche Wertschöpfung betrachtet. Diese wird dann geschaffen oder zerstört, wenn das individuelle Erleben und Verhalten von Personen und Gruppen so beeinflusst wird, dass dies stabilisierend oder destabilisierend auf Bewertungen des gesellschaftlichen Zusammenhalts, das Gemeinschaftserleben und die Selbstbestimmung des Einzelnen wirkt
(Meynhardt 2008). Vor dem Hintergrund einer „moralisierten“ und „beschleunigten“ Gesellschaft ist anzunehmen, dass Bereiche wie Mobilität, Daseinsvorsorge oder Gesundheit - und damit auch die entsprechenden Produkte und Dienstleistungen , prinzipiell nicht „außer Streit gestellt“ werden können. Insofern stehen Customer Value und Public Value in einem engen Wechselverhältnis.

Eine vielversprechende Konzeption zum Umgang mit daraus erwachsenden Anforderungen liegt in der Weiterentwicklung von der „Nutzen- zur Funktionenorientie- 
rung“ des Marketing von Haller (2004). Die Nutzenbetrachtung als Einschätzung des Kunden mit Blick auf das Potenzial einer Leistung zur Bedürfnisbefriedigung wird dabei ergänzt um „das Denken in Funktionen“ für den Kunden. Dies bietet sich vor allem an, wenn der Wettbewerb nicht allein auf der Ebene des Kernprodukts, sondern auf der Ebene einzelner Kern- und Zusatzfunktionen für den Kunden stattfindet. Neben den technisch-leistungswirtschaftlichen Funktionen (z. B. Service, Convenience etc.) können dies vor allem sozial-psychologische Funktionen (z. B. identitätsstiftende Mitgliedschaften in Gemeinschaften) sein.

In der Fortführung dieses Ansatzes rückt die „Funktion“ der Leistungen und Produkte aus Sicht der Gesellschaft in den Vordergrund. Der Blick wird nicht allein auf den Kunden im Marktumfeld, sondern auf positive wie negative Wirkungen auf Dritte, einzelne Gruppen und die Gesellschaft als Ganzes gerichtet. Dabei geht es nicht um die selbstverständliche Einhaltung von rechtlichen Rahmenbedingungen, sondern um die Wechselwirkung mit unterschiedlichen überindividuellen Bezugsebenen (z. B. Communities, Öffentlichkeit, Staat, Medien) und damit eine „Public Value Proposition“, welche den Legitimationshintergrund für eine „Customer Value Proposition" bildet.
Die Wirkungszusammenhänge des Marketing sind vor diesem Hintergrund nicht auf einen Customer Value als Wert für oder von den Kunden zu beschränken, sondern in übergreifenden Systembezügen zu verorten. Jeder Kundennutzen schafft oder vernichtet gesellschaftlichen Nutzen und steht damit in Beziehung zu Public Values. Wenn ein Unternehmen zwar Nutzen für die Kunden stiftet, aber Schaden für alle anderen verursacht, gerät es in Konflikt mit „Public Values“ und riskiert den Verlust seiner „Licence to Operate“ als „gesellschaftliche Geschäftsgrundlage“.

\section{Grundbedürfnisse als Bindeglied zwischen Customer Value und Public Value}

Es ist äußerst anspruchsvoll, im Kunden die Gesellschaft mitzubedenken. Ein wesentlicher Baustein ist die Reflexion und Gestaltung des Verhältnisses zwischen menschlichen Grundbedürfnissen und kollektiv verankerten „Werten“. Letztere haben eine kollektive Qualität, wenn sie von mehreren so verinnerlicht und damit geteilt werden, dass sie für diese Personen oder Gruppen handlungsleitend sind. Entscheidend ist aber auch hier, dass sie nur in den subjektiven Bewertungen des Einzelnen „existieren“. Sie finden ihren Ausdruck in Emotionen, Einstellungen, Haltungen, Präferenzen, Motivationen, Interes- sen, Meinungen, Vermutungen usw. (Meynhardt 2004).

Jeder subjektiv erlebte Kundennutzen kann als positive Bewertung einer komplexen Bedürfnisbefriedigung angesehen werden. Aus der psychologischen Theoriebildung können nun Annahmen über Bedürfnisse als Grundlagen von Bewertungsprozessen getroffen werden. „Bedürfnisse“ werden verstanden als bestimmte Mangelzustände, Bedarfe oder Defizite, welche emotional durch Zustände des Unwohlseins, der Bedrohung, der Anspannung usw. charakterisiert sind. Bedürfnisbefriedigung geht mit entsprechend emotional positiv getönten Zuständen einher (Kroeber-Riel/Weinberg 2003).

Nach Sichtung und Zusammenführung der bisherigen Theoriebildung lassen sich vier stabile Bedürfnisse ausmachen (Meynhardt 2004). Die Ergebnisse entsprechender Bewertungsprozesse sind analog strukturiert, wie Tabelle 1 verdeutlicht.

Je nach Blickwinkel kann die individuelle Bedürfnisbefriedigung im Sinne des Customer Value oder die gesellschaftliche Wertung (Public Value) im Vordergrund stehen. In einer Gemengelage unterschiedlichster, oftmals konflikthafter, sich schnell verändernder Bedürfnis- und Wertstrukturen ist daher die eigene Position zur „Natur“ des Menschen anhand von empirisch abgesicherten Kriterien zu reflektieren. Aufgrund des Wechselspiels

Tab. 2 Customer Value und Fragen aus einer Public-Value-Perspektive

\begin{tabular}{|c|c|c|c|c|}
\hline \multirow[b]{2}{*}{$\begin{array}{l}\text { Customer Value } \\
\text { (Kundenfokus) }\end{array}$} & \multicolumn{4}{|c|}{ Public Value-Funktionen (Gesellschaftsfokus) } \\
\hline & Ethisch-moralisch & Politisch-sozial & Hedonistisch-ästhetisch & $\begin{array}{l}\text { Instrumentell- } \\
\text { utilitaristisch }\end{array}$ \\
\hline Emotion & \multirow{10}{*}{$\begin{array}{l}\text { - Welche Art der Anerkennung } \\
\text { und Wertschätzung für das } \\
\text { Selbstwertempfinden/Identität } \\
\text { wollen wir über den Customer } \\
\text { Value erreichen? } \\
\text { - Wieviel Eigenverantwortung } \\
\text { wollen wir durch den Customer } \\
\text { Value dem einzelnen Kunden } \\
\text { abnehmen bzw. zuschreiben? } \\
\text { - Wie begründen wir im gesell- } \\
\text { schaftlichen Umfeld moralisch- } \\
\text { ethisch einen differenzierten } \\
\text { Umgang mit den Kunden? }\end{array}$} & \multirow{10}{*}{$\begin{array}{l}\text { - Welche Kultur des zwischen- } \\
\text { menschlichen Umgangs wollen } \\
\text { wir in der Realisierung des } \\
\text { Customer Value fördern? } \\
\text { - Welche Interessen einzelner } \\
\text { Gruppen bzw. Akteure in der } \\
\text { Gesellschaft werden durch den } \\
\text { Customer Value gestärkt/ } \\
\text { geschwächt? } \\
\text { Welche Art von Auseinander- } \\
\text { setzung des gesellschaftspoli- } \\
\text { tischen Dialogs zur Durch- } \\
\text { setzung einzelner Customer } \\
\text { Values wollen wir führen? }\end{array}$} & \multirow{10}{*}{$\begin{array}{l}\text { Welche negativen Erfahrungen } \\
\text { wollen wir durch jeden } \\
\text { einzelnen Customer Value in } \\
\text { Bezug auf unser Unternehmen } \\
\text { vermeiden? } \\
\text { - Welche positiven Erfahrungen } \\
\text { wollen wir durch jeden } \\
\text { einzelnen Customer Value in } \\
\text { Bezug auf unser Unternehmen } \\
\text { fördern? } \\
\text { Welche Erfahrungen bei Nicht- } \\
\text { Kunden streben wir als Unter- } \\
\text { nehmen an bzw. wollen wir } \\
\text { vermeiden? }\end{array}$} & \multirow{10}{*}{$\begin{array}{l}\text { - Welche sachlich-inhaltliche } \\
\text { Basis hat jeder einzelne } \\
\text { Customer Value? } \\
\text { - Welche Art von „Hilfe zur Selbst- } \\
\text { hilfe” wollen wir anbieten? } \\
\text { - Welche Standards der Problem- } \\
\text { lösung wollen wir durch jeden } \\
\text { Customer Value setzen und } \\
\text { prägen? }\end{array}$} \\
\hline Beziehung & & & & \\
\hline Erklärung & & & & \\
\hline Individualisierung & & & & \\
\hline Entlastung/Sicherheit & & & & \\
\hline Qualität & & & & \\
\hline Innovation & & & & \\
\hline Wirtschaftlichkeit & & & & \\
\hline Geschwindigkeit & & & & \\
\hline Koordination & & & & \\
\hline
\end{tabular}


von Customer Value und Public Value kann so in der Vielfalt und Fülle von Marketingansätzen und differenzierten Kundensegmentierungen immer wieder die eigene „Value Proposition“ reflektiert und wetterfest gemacht werden.

Public Values können nicht beliebig „zugekauft“ werden. Eine wesentliche Frage ist dabei die Konsistenz und Kohärenz zwischen der Value Proposition gegenüber Kunden und der übergreifenden Unternehmensstrategie. Im Mission Statement des Unternehmens sollten seine Core Values („Guiding Principles“) und sein Core Purpose („Reason for Being“) definiert sein. Dies zeigt sich etwa in der Übersetzung von Kundenvorteilen in Vorund Nachteilen für das gesellschaftliche Umfeld. In Tabelle 2 sind beispielhaft Fragen aus Sicht einer PublicValue-Perspektive aufgelistet, anhand derer die Kriterien der Konfiguration von Kundennutzen (Belz/Bieger 2004, S. 101) einzeln oder im Verbund systematisch reflektiert werden können.

Auf diese Weise sollte zum Beispiel ein angestrebter Customer Value „Geschwindigkeit“ entlang der vier Bewertungskriterien daraufhin reflektiert werden, warum der Kunde „beweglich und flexibel“ bleiben soll. In dieser Art der gesellschaftlichen Rückbindung des Customer Value in übergreifende Systembezüge sehen wir einen Weg, die wahrgenommene Komplexität angemessen zu vereinfachen.

Die in einer „Value Proposition“ zusammengeführten Antworten konkretisieren die Verkopplung von Customer Value und Public Value. Sie zielen auf die psychologischen und sozial-psychologischen Erfahrungsangebote durch Produkte und Dienstleistungen. Es ist unrealistisch, in allen Dimensionen ein harmonisches oder konfliktfreies Bild zu erreichen - vielmehr sind die erforderlichen Abwägungen zu reflektieren und bewusst zu treffen. In diesem Sinne wird es nicht ausreichen, wenn zum Beispiel ein Sportwagenhersteller nur auf den mit seinen Produkten verbundenen „Fahrspass“ abhebt. Eine „License to Operate" ließe sich vielleicht eher mit den positiven Sicherheits- und Umwelteffekten einer überlegenen Fahrzeugtechnik untermauern.

\section{Resümee}

Die Proliferation von Kundensegmenten, -bedürfnissen und -interaktionspunkten bedingt die immer stärkere Ausdifferenzierung der Customer Value Proposition. Zugleich bietet die Tendenz der sozialen Beschleunigung die Chance, in der Erfahrungswelt des Kunden sinnstiftend zu wirken. Demgegenüber steht die zunehmende Moralisierung der Märkte - Unternehmen können nicht nur, sie müssen sich sogar der Frage stellen, inwieweit ihr Handeln einen Wert für die Gemeinschaft hat. Da die ausdifferenzierte Betrachtung sämtlicher Stakeholder allerdings kaum praktikabel wäre, ist der abstraktere Bezug auf eine gesellschaftliche Ebene vorzuziehen: die „Wert“-Schöpfung für die Gemeinschaft legitimiert zum einen die unternehmerische Aktivität im Sinne einer gesellschaftlichen Geschäftsgrundlage. Zum anderen dient sie als Fundament für die vielfältig differenzierten Ausprägungen des Customer Value. Denn so wie der Kommunikationsforscher Paul Watzlawick formuliert, dass man nicht nicht kommunizieren kann, gilt eben auch: Man kann nicht nicht gesellschaftlich handeln. Jede Handlung hat Konsequenzen für den Einzelnen und die Gemeinschaft. Aus unserer Sicht sind hier auch innovative Instrumente gefordert. Aber im Kern geht es vor allem um eine intensivere Beschäftigung mit der Frage, was die eigenen Produkte und Dienstleistungen wertvoll für die Gesellschaft macht. Hier ist eine Public Value Proposition gefragt, die zuallererst eine entschlossene und mutige Positionierung beinhaltet. Diese hängt weniger von neuen „Tools“ als von einem verinnerlichten „Wertekompass“ ab. Der Customer Value hat im Public Value keinen Gegenspieler, sondern einen Mitspieler und vielleicht manchmal auch einen Schiedsrichter. In einer hoch vernetzten Gesellschaft kann überlegener Customer Value nur vor dem Hintergrund des Public Value generiert werden.

\section{Literatur}

Belz, Ch./Bieger Th. (Hrsg.) (2004): Customer Value - Kundenvorteile schaffen Unternehmensvorteile, Frankfurt am Main.

Court, David (2006): Profiting from Proliferation, New York.

Economist (2008): Beware grannies on Facebook, 17.04.2008.

Freeman, R.E. (1984): Strategic Management: A Stakeholder Approach, Boston.

Gomez, P./Probst, G. (1999): Die Praxis des ganzheitlichen Problemlösens: vernetzt denken, unternehmerisch handeln, persönlich überzeugen, Bern.

Haller, M. (2004): Funktionen-Ansatz, in: Belz, Ch./Bieger, Th. (Hrsg): Customer Value Kundenvorteile schaffen Unternehmensvorteile, Frankfurt am Main, S. 720-734.

Harrison, R., Shaw, D. \& Newholm, T. (Eds.) (2005): The Ethical Consumer, London.

Köcher, R. (2006): Die Distanz zwischen Bürgern und Wirtschaft wächst. Die Mehrheit glaubt nicht an einen Gewinn aus den Erfolgen der Unternehmen, in FAZ, 20.12.

Kotler, Ph. (2000): Marketing Management, Upper Saddle River.

Kroeber-Riel, W., Weinberg, P. (2003): Konsumentenverhalten, 8. Aufl., München.

Meffert, H. (2007): Marketing, 10. Aufl., Wiesbaden.

Meynhardt, T. (2004): Wertwissen: Was Organisationen wirklich bewegt, Münster.

Meynhardt, T. (2008): Public Value - oder: Was heißt Wertschöpfung zum Gemeinwohl?, in: der moderne staat, 2 .

Meynhardt, T. (in press): Public Value inside: What is public value creation?

Rosa, H. (2005): Beschleunigung - Die Veränderung der Zeitstrukturen in der Moderne, Frankfurt am Main.

Stehr, N. (2007): Die Moralisierung der Märkte: Eine Gesellschaftstheorie, Frankfurt am Main.

Walsh, J. P. (2005): Taking Stock of Stakeholder Management, Academy of Management Review, 30, 2, S. 426-452.

\section{Autoren}

Dr. Timo Meynhardt

Managing Director des Center for Leadership and Values in Society an der Universität St. Gallen.

E-Mail: timo.meynhardt@unisg.ch

Dr. Remmert A. Stock

Head of Group Corporate Development bei der Hypo Real Estate Holding AG, München. E-Mail: remmert.stock@hyporealestate.com 